ratio of Apo B/Apo A1 was also the highest than the norm. Therefore, we can assume that dyslipidemic disorders, without of effective means of correction, will contribute to the progression of both - $\mathrm{CP}$ and $\mathrm{AH}$.

\section{P336 HYPOALBUMINEMIA AND OEDEMA IN A 4 MONTH-OLD BOY}

${ }^{1}$ Deirdre O'Sullivan*, ${ }^{1}$ Rincy Koshy, Declan Cody 1,2, 1,2Billy Bourke. 'Our Lady's Children Hospital, Crumlin, Dublin, Ireland; ${ }^{2}$ University College Dublin, Dublin, Ireland

\subsection{6/archdischild-2019-epa.685}

Aims Primary intestinal lymphangiectasia (PIL) is a well-recognized congenital abnormality of the lymphatic system leading to protein-losing enteropathy (PLE). It is an uncommon disorder seldom seen in clinical practice. The prevalence is unknown. The objective of this case report is to describe the clinical presentation and diagnosis of PIL.

Methods A detailed chart review was performed. Data extracted from the medical records included presenting complaint, disease progression, laboratory results, imaging and clinical measurements.

Results The patient was a 4 month old boy, who presented to the emergency department in Our Lady's Children Hospital, Crumlin (OLCHC) with a 3 week history of bilateral lower limb swelling, easy bruising and a change in stool consistency. The baby was passing watery secretions prior to stool. Stool consistency was clay- like with mucous. Initial bloods showed a severe coagulopathy (PT 204.6 secs, APTT 80.4 secs), severe electrolyte disturbances $(\mathrm{Na}+128 \mathrm{mmol} / \mathrm{l}, \mathrm{K}+2.6 \mathrm{mmol} / \mathrm{l})$, mild transaminase elevation (AST $87 \mathrm{U} / 1$, ALT $49 \mathrm{U} / \mathrm{l}$ ), metabolic acidosis ( $\mathrm{pH} 7.293$, pCO2 4.75, HCO3- 16.9) and profound hypoalbuminemia $(13 \mathrm{~g} / \mathrm{l})$. The patient was admitted for electrolyte replacement and for further investigation of the underlying cause of the hypoalbuminemia and oedema. Stool alpha- 1-antitrypsin $(5.66 \mathrm{mg} / \mathrm{g})$ was markedly elevated confirming protein-losing enteropathy. Further investigations including endoscopic biopsies and allergy testing excluded other enteropathies such as coeliac disease, inflammatory bowel disease, congenital enteropathies and allergic enteritis. As in this case PIL pathology is often mid small bowel and beyond the reach of endoscopic biopsies.

Conclusion Hypoalbuminemia can manifestation from a variety of clinical disorders. This patient had an extensive work-up to exclude other possible causes. Once PLE was confirmed, further investigations were required to identify the underlying cause. The main laboratory findings that supported the diagnosis of PIL included hypoproteinaemia, protein losing enteropathy, lymphopenia, panhypogammaglobulinemia, malabsorption of fat-soluble vitamins and, ultimately, response to a low long chain triglyceride containing diet.

\section{P337 CURRENT PRACTICES FOR THE TREATMENT OF GASTROENTERITIS IN CHILDREN IN WEXFORD GENERAL HOSPITAL}

1,2Muhammad Zia*, 'Mohammad Taha, 'Maybelle wallis. 'Wexford general Hospital, wexford, Ireland; ${ }^{2}$ University Maternity Hospital Limerick, limerick, Ireland

10.1136/archdischild-2019-epa.686

Background Gastroenteritis(GE) is common in children.
Aim of the audit 1.To identify if rehydration treatment given was appropriate for the degree of dehydration?

2.To look how many children with gastroenteritis received saline boluses in emergency department or in ward when it was not indicated clinically.

3.To find out if we are using modes of rehydration other an intravenous in children with mild to moderate dehydration.

4.Whether ORS solution was used by parents before coming to hospital?

Methodology All children $<16$ years who presented to emergency department or were admitted in Gabriel ward with symptoms of diarrhoea or vomiting were included in the audit. The audit period was from $06 / 02 / 2018$ to 31/03/2018.

Results 1.Total 24 patients were collected from ED 20(83\%) and Gabriels ward4(17\%).

2. Out of them $11(45.8 \%)$ were female and 13(54.2\%)were male patients

3. Age ranges from 5 weeks to 11 years.13(54\%)patients were below 2 years of age.

4. All 24(100\%) patients had vomiting and $17(70.3 \%)$ patients had diarrhoea and vomiting at presentation.8(33.3\%) patients had temperature also.

5. $4(16.6 \%)$ patients had been given ORS before coming to hospital.

6. $18(75 \%)$ patients had no dehydration and 6(25\%) with clinical dehydration. No patients presented in shock.

7. Regarding mode of rehydration $20(83.3 \%)$ patients were given trial of oral rehydration and $4(16.7 \%)$ were given IV rehydration after failed oral challenge

8. IV bolus of Normal saline were given to $2(8.3 \%)$ patients.

9. Ondenstron was given to $18(75 \%)$ patients. $4(16.7 \%)$ patients failed oral challenge.

10. Nasogastric rehydration was not given to any of the patients.

Conclusions The use of ORS before coming to hospital was negligible. If used appropriately at home can reduce the hospital attendances. The NG mode of rehydration was not tried in any of our patients. By adapting NG mode of Rehydration we can avoid IV rehydration. The saline boluses were not indicated in patients who received them as they were not in severe dehydration or shock.

Recommendations and action plan 1. Education of GPS and parents about the importance of use of ORS once started with the symptoms of GE to avoid hospital admissions.

2. Awareness among NCHDs and paediatric nurses to use alternate routes of rehydration other than IV.

3. Patients presenting to ED department with no signs of dehydration should be encouraged for use of ORS at home.

4. Current practice of giving saline bolus in pateints who are not shocked should be discouraged.

\section{P338 COIN VS BATTERY - IMAGING OF FOREIGN BODIES}

Megan Sheppard*. Lady Cilento Children's Hospital , Brisbane , Australia

\subsection{6/archdischild-2019-epa.687}

A previously well 2 year old female presented to a peripheral emergency department following the ingestion of a foreign body. Her parents reported witnessing her swallow a coin, 2 days prior to presentation. She subsequently developed 24 hours of non-bilious vomiting followed by epigastric pain and 
decreased oral intake. The child was referred to a tertiary centre for review. On physical examination the child was alert but lethargic with tachycardia to 164 and a fever of 38.9 degrees. She had no drooling, increased work of breathing or stridor. Auscultation revealed equal air entry, normal heart and bowel sounds. Abdomen was soft, non tender without distension. She was treated with antipyretics and chest and abdominal imaging was arranged.

AP chest radiograph and plain abdominal images revealed a circular $2 \mathrm{~cm}$ radiopaque object located in the stomach. On review by radiology the object appeared to have a 'halo sign', a feature consistent with button batteries. Given the uncertain origin of the object and potential for complications associated with button battery ingestion our patient was fasted for an endoscopy.

Endoscopy revealed two 10 cent coins sitting in the gastric antrum with localized erosions and erythema. These were successfully removed with a Roth net and the patient was discharged the same day.

Foreign body (FB) ingestion/inhalation most commonly occurs in children aged between six months and three years. The most common objects are coins, reported in up to $88 \%$ of cases. (1) FB ingestion usually only requires imaging followed by clinical observation. Coins are unlikely to cause complications, whereas button batteries pose a risk of metal poisoning, burns, oesophgeal strictures, perforation, tracheooesophgeal and aorto-oesophgeal fistula and haemorrhage.(2) One study revealed that $14 \%$ of children with oesophageal FBs were asymptomatic on presentation, highlighting the need for imaging. Recommended investigations include AP and lateral neck, AP and lateral chest and abdominal radiographs. (4)

The sensitivity of plan films in detecting a battery was $80.4 \%$, specificity $79.1 \%$ with an overall accuracy of $79.8 \%$. The same study demonstrated that stacking two coins on top of each other lead to high rates of misidentification as batteries with an overall accuracy below 60\%. (1) Importantly this study used artificially produced images which therefore did not include soft tissues and bony structures which could prevent accurate identification.

This case highlights the importance of accurate identification of foreign objects and the need for high clinical suspicion of FB.

\section{P339 THROMBOCYTOPENIA IN CHILDREN WITH FEBRILE ILLNESS: CLINICAL COURSE AND OUTCOME}

${ }^{1}$ Ourania Alexandropoulou*, Lydia Kossiva ${ }^{2}$, ${ }^{3}$ Maria Giannaki, ${ }^{2}$ Maria Tsolia, ${ }^{2}$ Kyriaki Karavanaki. ' General Hospital, Agrinio, Greece; ${ }^{2}$ 2nd Department of Paediatrics, University of Athens, 'PandA Kyriakou' Children's Hospital, Athens, Greece; ${ }^{3}$ Microbiology Department, 'Aghia Sophia' Children's Hospital, Athens, Greece

10.1136/archdischild-2019-epa.688

Background Acquired thrombocytopenia in previously healthy children is common in paediatric practice and usually appears during infections.

Aim To assess the frequency, duration and clinical outcome of postinfectious thrombocytopenia in hospitalized febrile children.

Material-methods 117 febrile children with any kind of cytopenia aged $4.0 \pm 3.8$ years (range $0-14$ ), were admitted to a paediatric department during a 2-year period and were evaluated using inflammatory indices, cultures of body fluids and serological tests.
Results In 52/117 (44.4\%)cases a viral agent was identified. Among them 11/52 (21.1\%) had isolated thrombocytopenia, 32/52(61.5\%) had neutropenia/leukopenia and 9 $(17.3 \%)$ had thrombocytopenia with neutro/leukopenia or anemia.

The patients with viral infection had a mean $( \pm S D)$ age:3.3 \pm 3.9 years. The mean \pm SD duration of fever was $3.4 \pm 2.8$ days.

The highest prevalence of cytopenia was observed during spring (27/52) (51.9\%) and the lowest during fall (13.5\%).

Among the cases with thrombocytopenia, a viral agent was identified in $38.5 \%$ and a bacterial agent in $38.5 \%$.

In children with thrombocytopenia, the most frequently detected viruses were Epstein-Barr virus (10.2\%), followed by a herpesvirus $(6.1 \%)$. In cases with severe thrombocytopenia, mycoplasma infection was most frequent (4.1\%). Finally, in cases with bilineage cytopenia, influenza type A and B were more frequently detected among viruses, and Pseudomonas, Brucella and Rickettsia conorii among bacteria.

In cases of viral infections, thrombocytopenia was transient and lasted for $31.3 \pm 65.5$ days, while in those with 2 cell lines involvement (thrombocytopenia with neutropenia/leukopenia) it lasted for $26.8 \pm 38.9$ days.

In 13 cases with a bacterial infection $2 / 13$ (15.4\%) had thrombocytopenia and $3 / 13$ (23.1\%) thrombocytopenia with neutro/leukopenia.

Among the 21 cases with chronic cytopenia (11 girls and 10 boys), thrombocytopenia was present at 7/21 (33.3\%). Among them an infectious agent was identified in $2 / 7(28.6 \%)$ cases. One child with chronic thrombocytopenia was diagnosed having SLE.

In three children (two with thrombocytopenia and anemia and one with neutropenia/leukopenia and anemia), malignancy was finally diagnosed in a time-period of 4-7 months after the initial assessment. ( 2 with ALL and 1 with MDS).

Conclusion Postinfectious thrombocytopenia in childhood is usually transient with benign course, it resolves spontaneously and is usually associated with common viral or bacterial infections. However, in cases of chronic thrombocytopenia, especially if more than one cell lines are affected, close follow-up is necessary for the early detection of chronic hematologic or autoimmune disease.

\section{P340 CASE REPORT: IMMUNE THROMBOCYTOPENIA PURPURA (ITP) - AN INTRA-ORAL AND EXTRA-ORAL PRESENTATION}

Jennifer Maguire*, Kirsten Fitzgerald, Padraig Fleming. Our Lady's Children's Hospital Crumlin, Dublin, Ireland

10.1136/archdischild-2019-epa.689

A four year-old boy was referred to the Paediatric Dentistry Department by the Haematology Department at Our Lady's Children's Hospital Crumlin. He had a two week history of bleeding and crusting lips and intra-oral ulceration with a history of a cough two weeks prior to this. No relevant family history was noted.

The child's parents had initially presented him to community pharmacy and medical providers who advised attendance at the Emergency Department (ED) of the nearest tertiary care paediatric hospital. Upon presentation to the ED, he had a full blood count performed as part of his work up. Initial 